\title{
A basis for the right quantum algebra and the " $1=q$ " principle
}

\author{
Dominique Foata · Guo-Niu Han
}

Received: 16 January 2007 / Accepted: 21 May 2007 / Published online: 16 June 2007

(C) Springer Science+Business Media, LLC 2007

\begin{abstract}
We construct a basis for the right quantum algebra introduced by Garoufalidis, Lê and Zeilberger and give a method making it possible to go from an algebra subject to commutation relations (without the variable $q$ ) to the right quantum algebra by means of an appropriate weight-function. As a consequence, a strong quantum MacMahon Master Theorem is derived. Besides, the algebra of biwords is systematically in use.
\end{abstract}

Keywords Right quantum algebra · Quantum MacMahon master theorem

Mathematics Subject Classification (2000) Primary 05A19 - 05A30 - 16W35 • 17B37

\section{Introduction}

In their search for a natural q-analogue of the MacMahon Master Theorem, Garoufalidis et al. [4] have introduced the right quantum algebra $\mathcal{R}_{q}$ defined to be the associative algebra over a commutative ring $K$, generated by $r^{2}$ elements $X_{x a}(1 \leq x, a \leq r)$, $r \geq 2$, subject to the following commutation relations:

$$
\begin{aligned}
X_{y b} X_{x a}-X_{x a} X_{y b} & =q^{-1} X_{x b} X_{y a}-q X_{y a} X_{x b}, \quad x>y, a>b ; \\
X_{y a} X_{x a} & =q^{-1} X_{x a} X_{y a}, \quad x>y, \text { all } a
\end{aligned}
$$

D. Foata

Institut Lothaire, 1 rue Murner, 67000 Strasbourg, France

e-mail: foata@math.u-strasbg.fr

G.-N. Han ( $₫)$

I.R.M.A. UMR 7501, Université Louis Pasteur, 7 rue René-Descartes, 67084 Strasbourg, France e-mail: guoniu@math.u-strasbg.fr 
with $q$ being an invertible element in $K$. The right quantum algebra in the case $r=2$ has already been studied by Rodríguez-Romo and Taft [13], who set up an explicit basis for it. On the other hand, a basis for the full quantum algebra has been duly constructed (see [12, Theorem 3.5.1, p. 38]) for an arbitrary $r \geq 2$. It then seems natural to do the same with the right quantum algebra for each $r \geq 2$. This is the first goal of the paper.

In fact, the paper originated from a discussion with Doron Zeilberger, when he explained to the first author how he verified the quantum MacMahon Master identity for each fixed $r$ by computer code. His computer program uses the fact, as he is perfectly aware, that the set of irreducible biwords-which will be introduced in the sequel-generates the right quantum algebra. For a better understanding of his joint paper [4] and also for deriving the " $1=q$ " principle, it seems essential to see whether the set of irreducible biwords has the further property of being a basis, and it does. Thanks to this result, a strong quantum MacMahon Master Theorem can be subsequently derived.

When manipulating the above relations, the visible part of the commutations is made within the subcripts of the $X_{x a}$ 's, written as such. It is then important to magnify them by having an adequate notation. To this end, we replace each

$$
X_{x a} \text { by the biletter }\left(\begin{array}{l}
x \\
a
\end{array}\right) \text {, }
$$

so that, in further computations, products $X_{x_{1}, a_{1}} X_{x_{2}, a_{2}} \cdots X_{x_{\ell}, a_{\ell}}$ become biwords $\left(\begin{array}{l}x_{1} x_{2} \cdots x_{\ell} \\ a_{1} a_{2} \cdots a_{\ell}\end{array}\right)$, objects that have been efficiently used in combinatorial contexts $[9,10]$. Accordingly, the commutation rules for the right quantum algebra can be written as

$$
\begin{aligned}
\left(\begin{array}{l}
y x \\
b a
\end{array}\right)-\left(\begin{array}{l}
x y \\
a b
\end{array}\right) & =q^{-1}\left(\begin{array}{l}
x y \\
b a
\end{array}\right)-q\left(\begin{array}{l}
y x \\
a b
\end{array}\right), & x>y, a>b ; \\
\left(\begin{array}{l}
y x \\
a a
\end{array}\right) & =q^{-1}\left(\begin{array}{l}
x y \\
a a
\end{array}\right), & x>y, \text { all } a .
\end{aligned}
$$

We shall use the following notation. The positive integer $r$ will be kept fixed throughout and $\mathbb{A}$ will denote the alphabet $\{1,2, \ldots, r\}$. A biword on $\mathbb{A}$ is a $2 \times n$ matrix $\alpha=\left(\begin{array}{c}x_{1} \cdots x_{n} \\ a_{1} \cdots a_{n}\end{array}\right), n \geq 0$, whose entries are in $\mathbb{A}$, the first (resp. second) row being called the top word (resp. bottom word) of the biword $\alpha$. The number $n$ is the length of $\alpha$; we write $\ell(\alpha)=n$. The biword $\alpha$ can also be viewed as a word of biletters $\left(\begin{array}{l}x_{1} \\ a_{1}\end{array}\right) \cdots\left(\begin{array}{l}x_{n} \\ a_{n}\end{array}\right)$, those biletters $\left(\begin{array}{l}x_{i} \\ a_{i}\end{array}\right)$ being pairs of integers written vertically with $x_{i}, a_{i} \in \mathbb{A}$ for all $i=1, \ldots, n$. The product of two biwords is their concatenation.

Let $\mathbb{B}$ denote the set of biletters and $\mathcal{B}$ the set of all biwords. Let $\alpha \in \mathcal{B}$ such that $\ell(\alpha) \geq 2$ and $1 \leq i \leq \ell(\alpha)-1$. The biword $\alpha$ can be factorized as $\alpha=\beta\left(\begin{array}{l}x y \\ a b\end{array}\right) \gamma$, where $x, y, a, b \in \mathbb{A}$ and $\beta, \gamma \in \mathcal{B}$ with $\ell(\beta)=i-1$. Say that $\alpha$ has a double descent at position $i$ if $x>y$ and $a \geq b$. Notice the discrepancy in the inequalities on the top word and the bottom one. A biword $\alpha$ without any double descent is said to be irreducible. The set of all irreducible biwords is denoted by $\mathcal{B}_{\text {irr }}$.

Let $\mathbb{Z}$ be the ring of all integers, and let $\mathbb{Z}\left[q, q^{-1}\right]$ be the ring of the polynomials in the variables $q, q^{-1}$ subject to the rule $q q^{-1}=1$ with integral coefficients. The set $\mathcal{A}=\mathbb{Z}\langle\langle\mathcal{B}\rangle\rangle$ of the formal sums $\sum_{\alpha} c(\alpha) \alpha$, where $\alpha \in \mathcal{B}$ and $c(\alpha) \in \mathbb{Z}$, together with the above biword multiplication, the free addition and the free scalar product, forms an algebra over $\mathbb{Z}$, called the free biword large $\mathbb{Z}$-algebra. The formal sums 
$\sum_{\alpha} c(\alpha) \alpha$ will be called expressions. An expression $\sum_{\alpha} c(\alpha) \alpha$ is said to be irreducible if $c(\alpha)=0$ for all $\alpha \notin \mathcal{B}_{\text {irr }}$. The set of all irreducible expressions is denoted by $\mathcal{A}_{\text {irr }}$.

Similarly, let $\mathcal{A}_{q}=\mathbb{Z}\left[q, q^{-1}\right]\langle\langle\mathcal{B}\rangle\rangle$ denote the large $\mathbb{Z}\left[q, q^{-1}\right]$-algebra of the formal sums $\sum_{\alpha} c(\alpha) \alpha$, where $c(\alpha) \in \mathbb{Z}\left[q, q^{-1}\right]$ for all $\alpha \in \mathcal{B}$. Following Bergman's method [1] we introduce two reduction systems $(S)$ and $\left(S_{q}\right)$ as being the sets of pairs $(\alpha,[\alpha]) \in \mathcal{B} \times \mathcal{A}$ and $\left(\alpha,[\alpha]_{q}\right) \in \mathcal{B} \times \mathcal{A}_{q}$, respectively, defined by

$$
\begin{array}{ll}
\left(\left(\begin{array}{l}
x y \\
a b
\end{array}\right),\left[\begin{array}{l}
x y \\
a b
\end{array}\right]\right) \text { with }\left[\begin{array}{l}
x y \\
a b
\end{array}\right]=\left(\begin{array}{l}
y x \\
b a
\end{array}\right)+\left(\begin{array}{l}
y x \\
a b
\end{array}\right)-\left(\begin{array}{l}
x y \\
b a
\end{array}\right), & x>y, a>b ; \\
\left(\left(\begin{array}{l}
x y \\
a a
\end{array}\right),\left[\begin{array}{l}
x y \\
a b
\end{array}\right]\right) \text { with }\left[\begin{array}{l}
x y \\
a a
\end{array}\right]=\left(\begin{array}{l}
y x \\
a a
\end{array}\right), & x>y, \text { all } a .
\end{array}
$$

$$
\left(\left(\begin{array}{l}
x y \\
a b
\end{array}\right),\left[\begin{array}{l}
x y \\
a b
\end{array}\right]_{q}\right) \text { with }\left[\begin{array}{l}
x y \\
a b
\end{array}\right]_{q}=\left(\begin{array}{l}
y x \\
b a
\end{array}\right)+q\left(\begin{array}{l}
y x \\
a b
\end{array}\right)-q^{-1}\left(\begin{array}{l}
x y \\
b a
\end{array}\right), \quad x>y, a>b ;
$$

$$
\left(\left(\begin{array}{c}
x y \\
a a
\end{array}\right),\left[\begin{array}{l}
x y \\
a a
\end{array}\right]_{q}\right) \text { with }\left[\begin{array}{c}
x y \\
a a
\end{array}\right]_{q}=q\left(\begin{array}{c}
y x \\
a a
\end{array}\right), \quad x>y, \text { all } a .
$$

Notice that the equations $\left(\begin{array}{c}x y \\ a b\end{array}\right)-\left[\begin{array}{c}x y \\ a b\end{array}\right]_{q}=0,\left(\begin{array}{c}x y \\ a a\end{array}\right)-\left[\begin{array}{c}x y \\ a a\end{array}\right]_{q}=0$ are simple rewritings of the commutation rules of the right quantum algebra and that $(S)$ is deduced from $\left(S_{q}\right)$ by letting $q=1$.

Let $\mathcal{I}$ (resp. $\mathcal{I}_{q}$ ) be the two-sided ideal of $\mathcal{A}$ (resp. of $\mathcal{A}_{q}$ ) generated by the elements $\gamma-[\gamma]$ (resp. $\gamma-[\gamma]_{q}$ ) such that $(\gamma,[\gamma]) \in(S)$ (resp. $\gamma-[\gamma]_{q} \in\left(S_{q}\right)$ ). The quotient algebras $\mathcal{R}=\mathcal{A} / \mathcal{I}$ and $\mathcal{R}_{q}=\mathcal{A}_{q} / \mathcal{I}_{q}$ are called the 1-right quantum algebra and the $q$-right quantum algebra, respectively.

In Sect. 2 we define a $\mathbb{Z}$-linear mapping $E \mapsto[E]$ of $\mathcal{A}$ onto the $\mathbb{Z}$-module $\mathcal{A}_{\text {irr }}$ of the irreducible expressions, called reduction. Using Bergman's "Diamond Lemma" [1] this reduction will serve to obtain a model for the algebra $\mathcal{R}$, as stated in the next theorem.

Theorem 1 A set of representatives in $\mathcal{A}$ for $\mathcal{R}$ is given by the $\mathbb{Z}$-module $\mathcal{A}_{\text {irr }}$. The algebra $\mathcal{R}$ may be identified with the $\mathbb{Z}$-module $\mathcal{A}_{\text {irr }}$, the multiplication being given by $E \times F=[E \times F]$ for any two irreducible expressions $E, F$. With this identification $\mathcal{B}_{\text {irr }}$ is a basis for $\mathcal{R}$.

Four statistics counting various kinds of inversions will be needed. If $\alpha=\left(\begin{array}{l}u \\ v\end{array}\right)=$ $\left(\begin{array}{l}x_{1} x_{2} \cdots x_{n} \\ a_{1} a_{2} \cdots a_{n}\end{array}\right)$ is a biword, let

$$
\begin{aligned}
\operatorname{inv} u & =\#\left\{(i, j) \mid 1 \leq i<j \leq n, x_{i}>x_{j}\right\} ; \\
\operatorname{imv} v & =\#\left\{(i, j) \mid 1 \leq i<j \leq n, a_{i} \geq a_{j}\right\} ; \\
\operatorname{inv}^{-}(\alpha) & =\operatorname{inv} v-\operatorname{inv} u ; \\
\operatorname{inv}^{+}(\alpha) & =\operatorname{imv} v+\operatorname{inv} u .
\end{aligned}
$$

The first (resp. second) statistic "inv" (resp. "imv") is the usual number of inversions (resp. of large inversions) of a word. Notice that "inv" " may be negative. The weight function $\phi$ defined for each biword $\alpha$ by $\phi(\alpha)=q^{\text {inv }^{-}(\alpha)} \alpha$ can be extended to all of $\mathcal{A}_{q}$ by linearity. Clearly, it is a $\mathbb{Z}\left[q, q^{-1}\right]$-module isomorphism of $\mathcal{A}_{q}$ onto itself. The second result of the paper is stated next. 
Theorem 2 The $\mathbb{Z}\left[q, q^{-1}\right]$-module isomorphism $\phi$ of $\mathcal{A}_{q}$ onto itself induces a $\mathbb{Z}\left[q, q^{-1}\right]$-module isomorphism $\bar{\phi}$ of $\mathcal{A}_{q} / \mathcal{I}$ onto $\mathcal{A}_{q} / \mathcal{I}_{q}$. In particular, $\mathcal{R}_{q}$ has the same basis as $\mathcal{R}$.

Now, a circuit is defined to be a biword whose top word is a rearrangement of the letters of its bottom word. The set of all circuits is denoted by $\mathcal{C}$. It is clearly a submonoid of $\mathcal{B}$. An expression $E=\sum_{\alpha} c(\alpha) \alpha$ is said to be circular if $c(\alpha)=0$ for all $\alpha \notin \mathcal{C}$. Clearly, the sum and the product of two circular expressions is a circular expression, so that the set of circular expressions in $\mathcal{A}$ (resp. in $\mathcal{A}_{q}$ ) is a subalgebra of $\mathcal{A}$ (resp. of $\mathcal{A}_{q}$ ), which will be denoted by $\mathcal{A}^{\text {cir }}$ (resp. by $\mathcal{A}_{q}^{\text {cir }}$ ).

Theorem 3 The restriction of the $\mathbb{Z}\left[q, q^{-1}\right]$-module isomorphism $\bar{\phi}$ of Theorem 2 to $\mathcal{A}_{q}^{\mathrm{cir}} / \mathcal{I}$ is a $\mathbb{Z}\left[q, q^{-1}\right]$-algebra isomorphism onto $\mathcal{A}_{q}^{\mathrm{cir}} / \mathcal{I}_{q}$.
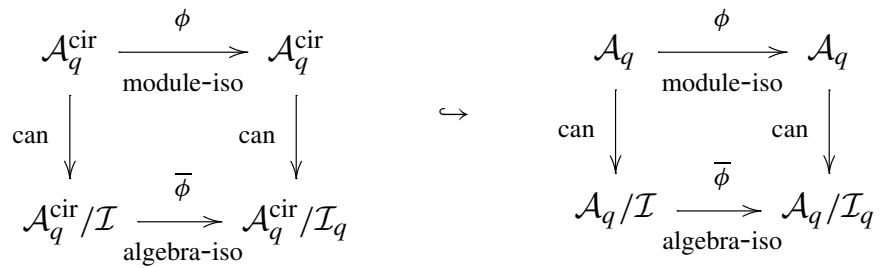

Accordingly, each identity holding in $\mathcal{A}_{q}^{\text {cir }} / \mathcal{I}$ has an equivalent counterpart in $\mathcal{A}_{q}^{\text {cir }} / \mathcal{I}_{q}$. This is the " $1=q$ " principle. An illustration of this principle is given by the $q \mathrm{MM}$ Theorem derived by Garoufalidis et al. [4]. Those authors have introduced the $q$-Fermion as being the sum

$$
\operatorname{Ferm}(q)=\sum_{J \subset \mathbb{A}}(-1)^{|J|} \sum_{\sigma \in \mathfrak{S}_{J}}(-q)^{-\operatorname{inv} \sigma}\left(\begin{array}{cccc}
\sigma\left(i_{1}\right) \sigma\left(i_{2}\right) & \cdots & \sigma\left(i_{l}\right) \\
i_{1} & i_{2} & \cdots & i_{l}
\end{array}\right),
$$

where $\mathfrak{S}_{J}$ is the permutation group acting on the set $J=\left\{i_{1}<i_{2}<\cdots<i_{l}\right\}$, and the $q$-Boson as the sum

$$
\operatorname{Bos}(q)=\sum_{w} q^{\operatorname{inv} w}\left(\begin{array}{l}
\bar{w} \\
w
\end{array}\right)
$$

over all words $w$ from the free monoid $\mathbb{A}^{*}$ generated by $\mathbb{A}$, where $\bar{w}$ stands for the nondecreasing rearrangement of the word $w$. The $q$-Fermion and $q$-Boson belong to $\mathcal{A}_{q}$. Garoufalidis et al. have proved ([4], see also [3] for another proof) the following identity

$$
\operatorname{Ferm}(q) \times \operatorname{Bos}(q) \equiv 1\left(\bmod \mathcal{I}_{q}\right) .
$$

This identity may a priori be regarded as a q-version of the MacMahon Master Theorem, as it reduces to the classical MacMahon's identity [11, pp. 93-98], when $q=1$ and the biletters are supposed to commute. By the " $1=q$ " principle (Theorem 3 ), we obtain the following result, which shows that the variable $q$ is in fact superfluous. 
Corollary 4 The identity $\operatorname{Ferm}(q) \times \operatorname{Bos}(q) \equiv 1\left(\bmod \mathcal{I}_{q}\right)$ holds if and only if $\operatorname{Ferm}(1) \times \operatorname{Bos}(1) \equiv 1(\bmod \mathcal{I})$ holds.

When applying Theorem 1 to the quantum MacMahon Master Theorem, we obtain the following result, which can be regarded as a strong quantum MacMahon Master Theorem.

Corollary 5 The following identity holds:

$$
[\operatorname{Ferm}(1) \times \operatorname{Bos}(1)]=1
$$

The proof of Theorem 1, given in Sect. 2, is based on Bergman's “Diamond Lemma" [1, Theorem 1.2]. It consists of verifying that the conditions required by the "Diamond Lemma" hold in the present situation. Theorems 2 and 3 are proved in Sect. 3 and Corollaries 4 and 5 in the last section.

\section{The reduction process}

For applying Bergman's "Diamond Lemma" [1] we need a so-called reduction system that satisfies two conditions: (1) the descending chain condition holds; (2) its ambiguities are resolvable. Those terms will be explained shortly. Let $\mathbb{Z}\langle\mathcal{B}\rangle$ be the subalgebra of $\mathcal{A}$ of all sums $\sum_{\alpha} c(\alpha) \alpha(\alpha \in \mathcal{B})$, where only a finite number of the $c(\alpha)$ 's are non-zero. The elements of $\mathbb{Z}\langle\mathcal{B}\rangle$ will be called finite expressions. The reduction system for $\mathbb{Z}\langle\mathcal{B}\rangle$ is simply the set $(S)$ of pairs $(\alpha,[\alpha]) \in \mathcal{B} \times \mathbb{Z}\langle\mathcal{B}\rangle$, already described in Sect. 1. By means of this reduction system we introduce the reduction itself as being the mapping $E \mapsto[E]$ of $\mathbb{Z}\langle\mathcal{B}\rangle$ onto the set $\mathbb{Z}\left\langle\mathcal{B}_{\text {irr }}\right\rangle$ of the finite irreducible expressions defined by the following axioms:

(C1) The reduction is linear, i.e. for $E_{1}, E_{2} \in \mathbb{Z}\langle\mathcal{B}\rangle$,

$$
\left[c_{1} E_{1}+c_{2} E_{2}\right]=c_{1}\left[E_{1}\right]+c_{2}\left[E_{2}\right] .
$$

(C2) $[E]=E$ for every $E \in \mathbb{Z}\left\langle\mathcal{B}_{\text {irr }}\right\rangle$.

(C3) For $\left(\begin{array}{l}x y \\ a b\end{array}\right) \notin \mathcal{B}_{\text {irr }}$, i.e. $x>y$ and $a \geq b$, then

$$
\begin{gathered}
{\left[\begin{array}{l}
x y \\
a b
\end{array}\right]=\left(\begin{array}{l}
y x \\
b a
\end{array}\right)+\left(\begin{array}{l}
y x \\
a b
\end{array}\right)-\left(\begin{array}{l}
x y \\
b a
\end{array}\right) \quad \text { if } a>b ;} \\
{\left[\begin{array}{l}
x y \\
a a
\end{array}\right]=\left(\begin{array}{l}
y x \\
a a
\end{array}\right) \quad \text { if } a=b .}
\end{gathered}
$$

(C4) Let $\alpha \notin \mathcal{B}_{\text {irr }}$ be a reducible biword. For each factorization $\alpha=\beta\left(\begin{array}{l}x y \\ a b\end{array}\right) \gamma$, where $x, y, a, b \in \mathbb{A}, \beta\left(\begin{array}{l}x \\ a\end{array}\right) \in \mathcal{B}_{\text {irr }}$ and $\gamma \in \mathcal{B}$, we have

$$
[\alpha]=\left[\beta \times\left[\begin{array}{l}
x y \\
a b
\end{array}\right] \times \gamma\right] .
$$

Although the reduction is recursively defined, it is well defined. Every time Condition (C3) is applied, the running biword $\alpha$ is transformed into either three new biwords (C3.1) or a new biword (C3.2). The important property is the fact that the 
statistic inv ${ }^{+}$of each of the new biwords is strictly less than $\operatorname{inv}^{+}(\alpha)$. Thus, after finitely many successive applications of (C3), an irreducible expression is derived. When the biword $\alpha$ has more than one double descent, Condition (C4) says that Condition (C3) must be applied at the first double descent position. Consequently, the final irreducible expression is unique.

There are several other ways to map each expression onto an irreducible expression, using relations (C3.1) and (C3.2). The reduction defined above is only one of those mappings. The important feature is the fact that such a mapping involves $f$ nitely many applications of relations (C3.1) and (C3.2). Following Bergman we say that Condition (1) (the descending chain condition) holds for the pair $(\mathbb{Z}\langle\mathcal{B}\rangle, S)$.

Now an expression $E$ is said to be reduction-unique under $S$ if the irreducible expression derived from $E$ does not depend on where the applications of (C3.1) and (C3.2) take place. More formally, each expression is reduction-unique under $S$ if for every biword $\alpha$ and every factorization $\alpha=\beta \alpha^{\prime} \gamma\left(\beta, \alpha^{\prime}, \gamma \in \mathcal{B}\right)$ we have

(Reduction-unique) $\quad[\alpha]=\left[\beta\left[\alpha^{\prime}\right] \gamma\right]$.

Now examine the second condition required by the "Diamond Lemma." There is an ambiguity in $S$ if there are two pairs $(\alpha,[\alpha])$ and $\left(\alpha^{\prime},\left[\alpha^{\prime}\right]\right)$ in $S$ such that $\alpha=$ $\beta \gamma, \alpha^{\prime}=\gamma \delta$ for some nonempty biwords $\beta, \gamma, \delta \in \mathcal{B}$. This ambiguity is said to be resolvable if $[[\alpha] \delta]=\left[\beta\left[\alpha^{\prime}\right]\right]$, i.e. $[[\beta \gamma] \delta]=[\beta[\gamma \delta]]$. In our case, this means that $\beta=\left(\begin{array}{l}x \\ a\end{array}\right), \gamma=\left(\begin{array}{l}y \\ b\end{array}\right), \delta=\left(\begin{array}{l}z \\ c\end{array}\right)$ with $x>y>z$ and $a \geq b \geq c$. Using the first three integers instead of the letters $x, y, z, a, b, c$ there are four cases to be studied:

(i) $\beta=\left(\begin{array}{l}3 \\ 3\end{array}\right), \gamma=\left(\begin{array}{l}2 \\ 2\end{array}\right), \delta=\left(\begin{array}{l}1 \\ 1\end{array}\right)$;

(ii) $\beta=\left(\begin{array}{l}3 \\ 2\end{array}\right), \gamma=\left(\begin{array}{l}2 \\ 2\end{array}\right), \delta=\left(\begin{array}{l}1 \\ 1\end{array}\right)$;

(iii) $\beta=\left(\begin{array}{l}3 \\ 2\end{array}\right), \gamma=\left(\begin{array}{l}2 \\ 1\end{array}\right), \delta=\left(\begin{array}{l}1 \\ 1\end{array}\right)$;

(iv) $\beta=\left(\begin{array}{l}3 \\ 1\end{array}\right), \gamma=\left(\begin{array}{l}2 \\ 1\end{array}\right), \delta=\left(\begin{array}{l}1 \\ 1\end{array}\right)$.

Accordingly, the ambiguities in $S$ are resolvable if the following four identities hold:

$$
\begin{aligned}
& {\left[\left[\begin{array}{l}
32 \\
32
\end{array}\right]\left(\begin{array}{l}
1 \\
1
\end{array}\right)\right]=\left[\left(\begin{array}{l}
3 \\
3
\end{array}\right)\left[\begin{array}{l}
21 \\
21
\end{array}\right]\right] ;} \\
& {\left[\left[\begin{array}{l}
32 \\
22
\end{array}\right]\left(\begin{array}{l}
1 \\
1
\end{array}\right)\right]=\left[\left(\begin{array}{l}
3 \\
2
\end{array}\right)\left[\begin{array}{l}
21 \\
21
\end{array}\right]\right] ;} \\
& {\left[\left[\begin{array}{l}
32 \\
21
\end{array}\right]\left(\begin{array}{l}
1 \\
1
\end{array}\right)\right]=\left[\left(\begin{array}{l}
3 \\
2
\end{array}\right)\left[\begin{array}{l}
21 \\
11
\end{array}\right]\right] ;} \\
& {\left[\left[\begin{array}{l}
32 \\
11
\end{array}\right]\left(\begin{array}{l}
1 \\
1
\end{array}\right)\right]=\left[\left(\begin{array}{l}
3 \\
1
\end{array}\right)\left[\begin{array}{l}
21 \\
11
\end{array}\right]\right],}
\end{aligned}
$$

Let us prove (2.1), the other three identities being handled in a similar manner.

Proof of (2.1) For an easy reading of the coming calculations we have added subscripts $A, B, \ldots, J$ to certain brackets, which should help spot the subscripted brackets in the various equations. The left-hand side is evaluated as follows:

$$
\left[\left[\begin{array}{l}
32 \\
32
\end{array}\right]\left(\begin{array}{l}
1 \\
1
\end{array}\right)\right]=\left[\left[\begin{array}{l}
23 \\
23
\end{array}\right]\left(\begin{array}{l}
1 \\
1
\end{array}\right)\right]_{A}+\left[\left[\begin{array}{l}
23 \\
32
\end{array}\right]\left(\begin{array}{l}
1 \\
1
\end{array}\right)\right]_{B}-\left[\left[\begin{array}{l}
32 \\
23
\end{array}\right]\left(\begin{array}{l}
1 \\
1
\end{array}\right)\right]_{C} ;
$$




$$
\begin{aligned}
& {\left[\left[\begin{array}{l}
23 \\
23
\end{array}\right]\left(\begin{array}{l}
1 \\
1
\end{array}\right)\right]_{A}=\left[\left(\begin{array}{l}
2 \\
2
\end{array}\right)\left[\begin{array}{l}
31 \\
31
\end{array}\right]\right]=\left[\left(\begin{array}{l}
2 \\
2
\end{array}\right)\left[\begin{array}{l}
13 \\
13
\end{array}\right]\right]_{D}+\left[\left(\begin{array}{l}
2 \\
2
\end{array}\right)\left[\begin{array}{l}
13 \\
31
\end{array}\right]\right]_{I}-\left[\left(\begin{array}{l}
2 \\
2
\end{array}\right)\left[\begin{array}{l}
31 \\
13
\end{array}\right]\right]_{J} ;} \\
& {\left[\left[\begin{array}{l}
23 \\
32
\end{array}\right]\left(\begin{array}{l}
1 \\
1
\end{array}\right)\right]_{B}=\left[\left(\begin{array}{l}
2 \\
3
\end{array}\right)\left[\begin{array}{l}
31 \\
21
\end{array}\right]\right]=\left[\left(\begin{array}{l}
2 \\
3
\end{array}\right)\left[\begin{array}{l}
13 \\
12
\end{array}\right]\right]_{E}+\left[\left(\begin{array}{l}
2 \\
3
\end{array}\right)\left[\begin{array}{l}
13 \\
21
\end{array}\right]\right]_{F}-\left[\left(\begin{array}{l}
2 \\
3
\end{array}\right)\left[\begin{array}{l}
31 \\
12
\end{array}\right]\right] ;} \\
& -\left[\left[\begin{array}{l}
32 \\
23
\end{array}\right]\left(\begin{array}{l}
1 \\
1
\end{array}\right)\right]_{C}=-\left[\left(\begin{array}{l}
3 \\
2
\end{array}\right)\left[\begin{array}{l}
21 \\
31
\end{array}\right]\right]=-\left[\left(\begin{array}{l}
3 \\
2
\end{array}\right)\left[\begin{array}{l}
12 \\
13
\end{array}\right]\right]_{G}-\left[\left(\begin{array}{l}
3 \\
2
\end{array}\right)\left[\begin{array}{l}
12 \\
31
\end{array}\right]\right]+\left[\left(\begin{array}{l}
3 \\
2
\end{array}\right)\left[\begin{array}{l}
21 \\
13
\end{array}\right]\right]_{H} ; \\
& {\left[\left(\begin{array}{l}
2 \\
2
\end{array}\right)\left[\begin{array}{l}
13 \\
13
\end{array}\right]\right]_{D}=\left[\left[\begin{array}{l}
21 \\
32
\end{array}\right]\left(\begin{array}{l}
3 \\
3
\end{array}\right)\right]=\left[\left[\begin{array}{l}
12 \\
12
\end{array}\right]\left(\begin{array}{l}
3 \\
3
\end{array}\right)\right]+\left[\left[\begin{array}{l}
12 \\
21
\end{array}\right]\left(\begin{array}{l}
3 \\
3
\end{array}\right)\right]-\left[\left[\begin{array}{l}
21 \\
12
\end{array}\right]\left(\begin{array}{l}
3 \\
3
\end{array}\right)\right] \text {; }} \\
& {\left[\left(\begin{array}{l}
2 \\
3
\end{array}\right)\left[\begin{array}{l}
13 \\
12
\end{array}\right]\right]_{E}=\left[\left[\begin{array}{l}
21 \\
31
\end{array}\right]\left(\begin{array}{l}
3 \\
2
\end{array}\right)\right]=\left[\left[\begin{array}{l}
12 \\
13
\end{array}\right]\left(\begin{array}{l}
3 \\
2
\end{array}\right)\right]+\left[\left[\begin{array}{l}
12 \\
31
\end{array}\right]\left(\begin{array}{l}
3 \\
2
\end{array}\right)\right]-\left[\left[\begin{array}{l}
21 \\
13
\end{array}\right]\left(\begin{array}{l}
3 \\
2
\end{array}\right)\right] \text {; }} \\
& {\left[\left(\begin{array}{l}
2 \\
3
\end{array}\right)\left[\begin{array}{l}
13 \\
21
\end{array}\right]\right]_{F}=\left[\left[\begin{array}{l}
21 \\
32
\end{array}\right]\left(\begin{array}{l}
3 \\
1
\end{array}\right)\right]=\left[\left[\begin{array}{l}
12 \\
23
\end{array}\right]\left(\begin{array}{l}
3 \\
1
\end{array}\right)\right]+\left[\left[\begin{array}{l}
12 \\
32
\end{array}\right]\left(\begin{array}{l}
3 \\
1
\end{array}\right)\right]-\left[\left[\begin{array}{l}
21 \\
23
\end{array}\right]\left(\begin{array}{l}
3 \\
1
\end{array}\right)\right]_{I} ;} \\
& -\left[\left(\begin{array}{l}
3 \\
2
\end{array}\right)\left[\begin{array}{l}
12 \\
13
\end{array}\right]\right]_{G}=-\left[\left[\begin{array}{l}
31 \\
21
\end{array}\right]\left(\begin{array}{l}
2 \\
3
\end{array}\right)\right]=-\left[\left[\begin{array}{l}
13 \\
12
\end{array}\right]\left(\begin{array}{l}
2 \\
3
\end{array}\right)\right]-\left[\left[\begin{array}{l}
13 \\
21
\end{array}\right]\left(\begin{array}{l}
2 \\
3
\end{array}\right)\right]+\left[\left[\begin{array}{l}
31 \\
12
\end{array}\right]\left(\begin{array}{l}
2 \\
3
\end{array}\right)\right] \text {; } \\
& {\left[\left(\begin{array}{l}
3 \\
2
\end{array}\right)\left[\begin{array}{l}
21 \\
13
\end{array}\right]\right]_{H}=\left[\left[\begin{array}{l}
32 \\
21
\end{array}\right]\left(\begin{array}{l}
1 \\
3
\end{array}\right)\right]=\left[\left[\begin{array}{l}
23 \\
12
\end{array}\right]\left(\begin{array}{l}
1 \\
3
\end{array}\right)\right]+\left[\left[\begin{array}{l}
23 \\
21
\end{array}\right]\left(\begin{array}{l}
1 \\
3
\end{array}\right)\right]_{J}-\left[\left[\begin{array}{l}
32 \\
12
\end{array}\right]\left(\begin{array}{l}
1 \\
3
\end{array}\right)\right] \text {. }}
\end{aligned}
$$

The sum of the above nine equalities yields

$$
\begin{aligned}
{\left[\left[\begin{array}{l}
32 \\
32
\end{array}\right]\left(\begin{array}{l}
1 \\
1
\end{array}\right)\right]=} & -\left(\begin{array}{l}
231 \\
312
\end{array}\right)-\left(\begin{array}{l}
312 \\
231
\end{array}\right)+\left(\begin{array}{l}
123 \\
123
\end{array}\right)+\left(\begin{array}{l}
123 \\
213
\end{array}\right)-\left(\begin{array}{l}
213 \\
123
\end{array}\right) \\
& +\left(\begin{array}{l}
123 \\
132
\end{array}\right)+\left(\begin{array}{l}
123 \\
312
\end{array}\right)-\left(\begin{array}{l}
213 \\
132
\end{array}\right)+\left(\begin{array}{l}
123 \\
231
\end{array}\right)+\left(\begin{array}{l}
123 \\
321
\end{array}\right) \\
& -\left(\begin{array}{l}
132 \\
123
\end{array}\right)-\left(\begin{array}{l}
132 \\
213
\end{array}\right)+\left(\begin{array}{l}
312 \\
123
\end{array}\right)+\left(\begin{array}{l}
231 \\
123
\end{array}\right)-\left(\begin{array}{l}
321 \\
123
\end{array}\right) .
\end{aligned}
$$

As for the right-hand side we have

$$
\begin{gathered}
{\left[\left(\begin{array}{l}
3 \\
3
\end{array}\right)\left[\begin{array}{l}
21 \\
21
\end{array}\right]\right]=\left[\left(\begin{array}{l}
3 \\
3
\end{array}\right)\left[\begin{array}{l}
12 \\
12
\end{array}\right]\right]_{A}+\left[\left(\begin{array}{l}
3 \\
3
\end{array}\right)\left[\begin{array}{l}
12 \\
21
\end{array}\right]\right]_{B}-\left[\left(\begin{array}{l}
3 \\
3
\end{array}\right)\left[\begin{array}{l}
21 \\
12
\end{array}\right]\right]_{C} ;} \\
{\left[\left[\begin{array}{l}
31 \\
31
\end{array}\right]\left(\begin{array}{l}
2 \\
2
\end{array}\right)\right]_{A}=\left[\left[\begin{array}{l}
13 \\
13
\end{array}\right]\left(\begin{array}{l}
2 \\
2
\end{array}\right)\right]_{D}+\left[\left[\begin{array}{l}
13 \\
31
\end{array}\right]\left(\begin{array}{l}
2 \\
2
\end{array}\right)\right]_{I}-\left[\left[\begin{array}{l}
31 \\
13
\end{array}\right]\left(\begin{array}{l}
2 \\
2
\end{array}\right)\right]_{J} ;} \\
{\left[\left[\begin{array}{l}
31 \\
32
\end{array}\right]\left(\begin{array}{l}
2 \\
1
\end{array}\right)\right]_{B}=\left[\left[\begin{array}{l}
13 \\
23
\end{array}\right]\left(\begin{array}{l}
2 \\
1
\end{array}\right)\right]_{E}+\left[\left[\begin{array}{l}
13 \\
32
\end{array}\right]\left(\begin{array}{l}
2 \\
1
\end{array}\right)\right]_{F}-\left[\left[\begin{array}{l}
31 \\
23
\end{array}\right]\left(\begin{array}{l}
2 \\
1
\end{array}\right)\right] ;} \\
-\left[\left[\begin{array}{l}
32 \\
31
\end{array}\right]\left(\begin{array}{l}
1 \\
2
\end{array}\right)\right]_{C}=-\left[\left[\begin{array}{l}
23 \\
13
\end{array}\right]\left(\begin{array}{l}
1 \\
2
\end{array}\right)\right]_{G}-\left[\left[\begin{array}{l}
23 \\
31
\end{array}\right]\left(\begin{array}{l}
1 \\
2
\end{array}\right)\right]+\left[\left[\begin{array}{l}
32 \\
13
\end{array}\right]\left(\begin{array}{l}
1 \\
2
\end{array}\right)\right]_{H} ; \\
{\left[\left(\begin{array}{l}
1 \\
1
\end{array}\right)\left[\begin{array}{l}
32 \\
32
\end{array}\right]\right]_{D}=\left[\left(\begin{array}{l}
1 \\
1
\end{array}\right)\left[\begin{array}{l}
23 \\
23
\end{array}\right]\right]+\left[\left(\begin{array}{l}
1 \\
1
\end{array}\right)\left[\begin{array}{l}
23 \\
32
\end{array}\right]\right]-\left[\left(\begin{array}{l}
1 \\
1
\end{array}\right)\left[\begin{array}{l}
32 \\
23
\end{array}\right]\right] ;} \\
{\left[\left(\begin{array}{l}
1 \\
2
\end{array}\right)\left[\begin{array}{l}
32 \\
31
\end{array}\right]\right]_{E}=\left[\left(\begin{array}{l}
1 \\
2
\end{array}\right)\left[\begin{array}{l}
23 \\
13
\end{array}\right]\right]+\left[\left(\begin{array}{l}
1 \\
2
\end{array}\right)\left[\begin{array}{l}
23 \\
31
\end{array}\right]\right]-\left[\left(\begin{array}{l}
1 \\
2
\end{array}\right)\left[\begin{array}{l}
32 \\
13
\end{array}\right]\right] ;} \\
{\left[\left(\begin{array}{l}
1 \\
3
\end{array}\right)\left[\begin{array}{l}
32 \\
21
\end{array}\right]\right]_{F}=\left[\left(\begin{array}{l}
1 \\
3
\end{array}\right)\left[\begin{array}{l}
23 \\
12
\end{array}\right]\right]+\left[\left(\begin{array}{l}
1 \\
3
\end{array}\right)\left[\begin{array}{l}
23 \\
21
\end{array}\right]\right]-\left[\left(\begin{array}{l}
1 \\
3
\end{array}\right)\left[\begin{array}{l}
32 \\
12
\end{array}\right]\right]} \\
-\left[\left(\begin{array}{l}
2 \\
1
\end{array}\right)\left[\begin{array}{l}
31 \\
32
\end{array}\right]\right]_{G}=-\left[\left(\begin{array}{l}
2 \\
1
\end{array}\right)\left[\begin{array}{l}
13 \\
23
\end{array}\right]\right]-\left[\left(\begin{array}{l}
2 \\
1
\end{array}\right)\left[\begin{array}{l}
13 \\
32
\end{array}\right]\right]+\left[\left(\begin{array}{l}
2 \\
1
\end{array}\right)\left[\begin{array}{l}
31 \\
23
\end{array}\right]\right] ; \\
{\left[\left(\begin{array}{l}
3 \\
1
\end{array}\right)\left[\begin{array}{l}
21 \\
32
\end{array}\right]\right]_{H}=\left[\left(\begin{array}{l}
3 \\
1
\end{array}\right)\left[\begin{array}{l}
12 \\
23
\end{array}\right]\right]+\left[\left(\begin{array}{l}
3 \\
1
\end{array}\right)\left[\begin{array}{l}
12 \\
32
\end{array}\right]\right]_{J}-\left[\left(\begin{array}{l}
3 \\
1
\end{array}\right)\left[\begin{array}{l}
21 \\
23
\end{array}\right]\right] .}
\end{gathered}
$$

The sum of the above nine equalities yields

$$
\begin{aligned}
{\left[\left(\begin{array}{l}
3 \\
3
\end{array}\right)\left[\begin{array}{l}
21 \\
21
\end{array}\right]\right]=} & -\left(\begin{array}{l}
312 \\
231
\end{array}\right)-\left(\begin{array}{l}
231 \\
312
\end{array}\right)+\left(\begin{array}{l}
123 \\
123
\end{array}\right)+\left(\begin{array}{l}
123 \\
132
\end{array}\right)-\left(\begin{array}{l}
132 \\
123
\end{array}\right) \\
& +\left(\begin{array}{l}
123 \\
213
\end{array}\right)+\left(\begin{array}{l}
123 \\
231
\end{array}\right)-\left(\begin{array}{l}
132 \\
213
\end{array}\right)+\left(\begin{array}{l}
123 \\
312
\end{array}\right)+\left(\begin{array}{l}
123 \\
321
\end{array}\right) \\
& -\left(\begin{array}{l}
213 \\
123
\end{array}\right)-\left(\begin{array}{l}
213 \\
132
\end{array}\right)+\left(\begin{array}{l}
231 \\
123
\end{array}\right)+\left(\begin{array}{l}
312 \\
123
\end{array}\right)-\left(\begin{array}{l}
321 \\
123
\end{array}\right) \\
= & {\left.\left[\begin{array}{l}
32 \\
32
\end{array}\right]\left(\begin{array}{l}
1 \\
1
\end{array}\right)\right] \text { (left-hand side) } . }
\end{aligned}
$$


Let $\mathcal{I}^{\prime}$ be the two-sided ideal of $\mathbb{Z}\langle\mathcal{B}\rangle$ generated by the elements $\gamma-[\gamma]$ such that $(\gamma,[\gamma]) \in(S)$, and let $\mathcal{R}^{\prime}$ be the quotient $\mathbb{Z}\langle\mathcal{B}\rangle / \mathcal{I}^{\prime}$. As the pair $(\mathbb{Z}\langle\mathcal{B}\rangle, S)$ satisfies the descending chain condition and since the ambiguities are resolvable, Bergman's "Diamond Lemma" implies the following theorem.

Theorem 6 A set of representatives in $\mathbb{Z}\langle\mathcal{B}\rangle$ for $\mathcal{R}^{\prime}$ is given by the $\mathbb{Z}$-module $\mathbb{Z}\left\langle\mathcal{B}_{\text {irr }}\right\rangle$. The algebra $\mathcal{R}^{\prime}$ may be identified with $\mathbb{Z}\left\langle\mathcal{B}_{\text {irr }}\right\rangle$, the multiplication being given by $E \times$ $F=[E \times F]$ for any two finite irreducible expressions $E, F$. With this identification $\mathcal{B}_{\text {irr }}$ is a basis for $\mathcal{R}^{\prime}$.

For the proof of Theorem 1 we use the following argument. For each $n \geq 0$ let $\mathcal{A}^{(n)}$ be the $n$-th degree homogeneous subspace of $\mathcal{A}$ consisting of all expressions $\sum_{\alpha} c(\alpha) \alpha$ such that $\ell(\alpha)=0$ if $\ell(\alpha) \neq n[6$, I.6]. As the starting alphabet $\mathbb{A}$ is finite, all expressions in $\mathcal{A}^{(n)}$ are finite sums, so that $\mathcal{A}^{(n)} \subset \mathbb{Z}\langle\mathcal{B}\rangle$ for all $n$. Now, let each expression $E$ from $\mathcal{A}$ be written as the sum

$$
E=\sum_{n \geq 0} E^{(n)} \quad \text { with } E^{(n)} \in \mathcal{A}^{(n)} .
$$

As both ideals $\mathcal{I}$ and $\mathcal{I}^{\prime}$ are generated by expressions from the second degree homogeneous space $\mathcal{A}^{(2)}$, we have $E \equiv 0(\bmod \mathcal{I})$ if and only if $E^{(n)} \equiv 0\left(\bmod \mathcal{I}^{\prime}\right)$ for every $n \geq 0$. In particular, $\left[E^{(n)}\right]$ also belongs to $\mathcal{A}^{(n)}$. Theorem 1 follows from Theorem 6 by taking the definition

$$
[E]=\sum_{n \geq 0}\left[E^{(n)}\right] .
$$

\section{The " $1=q$ " principle}

Let $E$ be an expression in $\mathcal{A}_{q}$. Theorem 2 is equivalent to saying that the identity $E \in \mathcal{I}$ holds if and only if $\phi(E) \in \mathcal{I}_{q}$. First, we prove the "only if" part. Because $\phi$ is linear, it suffices to consider all linear generators of $\mathcal{I}$, which have the following form:

$$
E_{1}=\alpha\left(\begin{array}{c}
r s \\
i i
\end{array}\right) \beta-\alpha\left(\begin{array}{c}
s r \\
i i
\end{array}\right) \beta
$$

and

$$
E_{2}=\alpha\left(\begin{array}{c}
r s \\
i j
\end{array}\right) \beta-\alpha\left(\begin{array}{c}
s r \\
j i
\end{array}\right) \beta-\alpha\left(\begin{array}{c}
s r \\
i j
\end{array}\right) \beta+\alpha\left(\begin{array}{c}
r s \\
j i
\end{array}\right) \beta,
$$

where $\alpha, \beta$ are biwords and $r, s, i, j$ are integers such that $r<s, i<j$. Let $k=$ inv $^{-}\left(\alpha\left(\begin{array}{c}r s \\ i j\end{array}\right) \beta\right)$, then

$$
\operatorname{inv}^{-}\left(\alpha\left(\begin{array}{c}
s r \\
j i
\end{array}\right) \beta\right)=k, \quad \operatorname{inv}^{-}\left(\alpha\left(\begin{array}{c}
s r \\
i j
\end{array}\right) \beta\right)=k-1, \quad \operatorname{inv}^{-}\left(\alpha\left(\begin{array}{c}
r s \\
j i
\end{array}\right) \beta\right)=k+1,
$$

so that

$$
\phi\left(E_{2}\right)=q^{k}\left(\alpha\left(\begin{array}{c}
r s \\
i j
\end{array}\right) \beta-\alpha\left(\begin{array}{c}
s r \\
j i
\end{array}\right) \beta-q^{-1} \alpha\left(\begin{array}{c}
s r \\
i j
\end{array}\right) \beta+q \alpha\left(\begin{array}{c}
r s \\
j i
\end{array}\right) \beta\right) \in \mathcal{I}_{q} .
$$

In the same way $\phi\left(E_{1}\right) \in \mathcal{I}_{q}$. The "if" part can be proved in the same manner. 
For Theorem 3 it suffices to prove the identity $\phi(E F)=\phi(E) \phi(F)$ for any two circular expressions $E$ and $F$. As $\phi$ is linear, it suffices to do it when $E=\left(\begin{array}{l}u \\ v\end{array}\right), F=$ $\left(\begin{array}{l}u^{\prime} \\ v^{\prime}\end{array}\right)$ are two circuits. Let $\operatorname{inv}\left(u, u^{\prime}\right)$ denote the number of pairs $(x, y)$ such that $x$ (resp. $y$ ) is a letter of $u$ (resp. of $u^{\prime}$ ) and $x>y$. Since $\left(\begin{array}{c}u u^{\prime} \\ v v^{\prime}\end{array}\right)$ is a circuit, we have

$$
\begin{aligned}
\operatorname{inv} u u^{\prime} & =\operatorname{inv} u+\operatorname{inv} u^{\prime}+\operatorname{inv}\left(u, u^{\prime}\right) ; \\
\operatorname{inv} v v^{\prime} & =\operatorname{inv} v+\operatorname{inv} v^{\prime}+\operatorname{inv}\left(v, v^{\prime}\right) ; \\
\operatorname{inv}\left(u, u^{\prime}\right) & =\operatorname{inv}\left(v, v^{\prime}\right) ;
\end{aligned}
$$

so that

$$
\begin{aligned}
\operatorname{inv}^{-}(E F) & =\operatorname{inv} v v^{\prime}-\operatorname{inv} u u^{\prime} \\
& =\operatorname{inv} v+\operatorname{inv} v^{\prime}-\operatorname{inv} u-\operatorname{inv} u^{\prime} \\
& =\operatorname{inv}^{-}(E)+\operatorname{inv}^{-}(F) .
\end{aligned}
$$

\section{The quantum MacMahon Master Theorem}

For proving Corollary 4 we apply Theorem 3 to Ferm(1) $\times$ Bos(1). Since Ferm(1) and $\operatorname{Bos}(1)$ are both circular expressions, the relation

$$
\operatorname{Ferm}(1) \times \operatorname{Bos}(1) \equiv 1(\bmod \mathcal{I})
$$

is equivalent to

$$
\phi(\operatorname{Ferm}(1)) \times \phi(\operatorname{Bos}(1)) \equiv 1\left(\bmod \mathcal{I}_{q}\right)
$$

Finally, it is straightforward to verify

$$
\operatorname{Ferm}(q)=\phi(\operatorname{Ferm}(1)) \quad \text { and } \quad \operatorname{Bos}(q)=\phi(\operatorname{Bos}(1)) \text {. }
$$

Now, to prove Corollary 5 we start with Garoufalidis et al.'s result (for $q=1$ ), which states that $\operatorname{Ferm}(1) \times \operatorname{Bos}(1) \equiv 1(\bmod \mathcal{I})$. But by Theorem 1 we have $E \equiv F(\bmod \mathcal{I})$ if and only if $[E]=[F]$. Hence $[\operatorname{Ferm}(1) \times \operatorname{Bos}(1)]=[1]=1$.

\section{Concluding remarks}

It is worth noticing that Rodríguez and Taft have introduced two explicit left quantum groups for $r=2$ in [13] and [14]. As mentioned in the introduction, the former one has been extended to an arbitrary dimension $r \geq 2$ by Garoufalidis et al. [4] and has been given an explicit basis in the present paper, while the latter one has been recently modeled after $\mathrm{SL}_{q}(r)$ by Lauve and Taft [8], also for each $r \geq 2$.

Since the paper has been submitted for publication, several works about the quantum MacMahon Master Theorem have been completed. Hai and Lorenz [5] have derived the identity using Koszul complex techniques. Konvalinka and Pak [7] have 
worked out a several basis $\left(q_{i j}\right)$-analogue of the quantum MacMahon Master Theorem and extended the " $1=q$ " principle developed in our paper to the case " $1=q_{i j}$ ". Etingof and Pak [2] have derived an extension taking up again the Koszul duality to obtain a new identity having several enumerative applications.

Acknowledgements We should like to thank Doron Zeilberger and Stavros Garoufalidis for several fruitful discussions. We are grateful to Christian Kassel, who gave us the references to the book by Parshall and Wang [12], the two papers by Rodríguez and Taft [13, 14] and the fundamental paper by Bergman [1]. Finally, we have greatly benefited from Jean-Pierre Jouanolou's ever-lasting mathematical expertise.

The authors should like to thank the two referees for careful reading and knowledgeable remarks.

\section{References}

1. Bergman, G. M. (1978). The diamond lemma for ring theory. Advances in Mathematics, 29, 178-218.

2. Etingof, P., \& Pak, I. An algebraic extension of the MacMahon Master Theorem. Proceedings of the American Mathematical Society, to appear.

3. Foata, D., \& Han, G.-N. (2007). A new proof of the Garoufalidis-Lê-Zeilberger quantum MacMahon Master Theorem. Journal of Algebra, 307, 424-431.

4. Garoufalidis, S., Lê, T.T.Q., \& Zeilberger, D. (2006). The quantum MacMahon Master Theorem. Proceedings of the National Academy of Science of the United States of America, 103, 13928-13931.

5. Hai, P. H., \& Lorenz, M. Koszul algebras and the quantum MacMahon Master Theorem. The Bulletin of the London Mathematical Society, to appear.

6. Kassel, C. (1995). Quantum groups. Graduate texts in mathematics (Vol. 155). New York: Springer.

7. Konvalinka, M., \& Pak, I. Non-commutative extensions of the MacMahon Master Theorem. Advances in Mathematics, to appear.

8. Lauve, A., \& Taft, E. J. (2007). A class of left quantum groups modeled after $S L_{q}(r)$. Journal of Pure and Applied Algebra, 208, 797-803.

9. Lothaire, M. (1983). Combinatorics on words. Encyclopedia of mathematics and its applications (Vol. 17). London: Addison-Wesley.

10. Lothaire, M. (2002). Algebraic combinatorics on words. Encyclopedia of mathematics and its applications (Vol. 90). Cambridge: Cambridge Univ. Press.

11. MacMahon, P. A. (1915). Combinatory analysis, Vol. 1. Cambridge Univ. Press. Reprinted by Chelsea Publ. Co., New York, 1960.

12. Parshall, B., \& Wang, J.-P. (1991). Quantum linear groups. Memoirs of the American Mathematical Society (Vol. 89).

13. Rodríguez-Romo, S., \& Taft, E. (2002). Some quantum-like Hopf algebras which remain noncommutative when $q=1$. Letters in Mathematical Physics, 61, 41-50.

14. Rodríguez-Romo, S., \& Taft, E. (2005). A left quantum group. Journal of Algebra, 286, 154-160. 